\title{
Processing without noticing in inattentional blindness: A replication of Moore and Egeth (1997) and Mack and Rock (1998)
}

\author{
Katherine Wood ${ }^{1}$ (D) Daniel J. Simons ${ }^{1}$
}

Published online: 19 November 2018

(C) The Psychonomic Society, Inc. 2018

\begin{abstract}
Surreptitious online measures can reveal the processing of stimuli that people do not report noticing or cannot describe. People seem to glean everything from low-level Gestalt grouping information to semantic meaning from unattended and unreported stimuli, and this information seems capable of influencing performance and of priming semantic judgments. Moore and Egeth (Journal of Experimental Psychology: Human Perception and Performance, 23, 339-352, 1997) provided evidence that judgments about the lengths of two lines were influenced by the grouping of background dots, even when subjects did not notice the pattern the dots formed. Mack and Rock (1998) reported that subjects could be primed to complete a stem with a word to which they were inattentionally blind. In this registered report, we replicated these two classic findings using large online samples $(N \mathrm{~s}=$ 260 and 448), finding support for the influence of grouping despite inattentional blindness, but not for word-stem priming.
\end{abstract}

Keywords Attention: Divided Attention and Inattention · implicit/explicit memory · attention

We experience visual perception as a seamless process-we open our eyes, light strikes our retinas, and we are aware of objects in the world. However, an object's reflected light reaching our eyes does not guarantee that we will notice the object: We can be inattentionally blind to objects in plain view if our attention is engaged elsewhere (e.g., Mack \& Rock, 1998; Neisser \& Becklen, 1975). What, if anything, do we absorb from these unnoticed and unreported objects?

Inattentional blindness (Mack, Tang, Tuma, Kahn, \& Rock, 1992), change blindness (Russell \& Driver, 2005), and motioninduced blindness (Mitroff \& Scholl, 2005) have all been used to present stimuli outside the focus of attention. In these paradigms, subjects do not report noticing stimuli and typically cannot select the features of those unnoticed stimuli at better-than-chance levels. The failure to notice coupled with a failure to identify features is often taken as evidence for the absence of awareness.

Electronic supplementary material The online version of this article (https://doi.org/10.3758/s13414-018-1629-1) contains supplementary material, which is available to authorized users.

Katherine Wood

kmwood2@illinois.edu

1 Department of Psychology, University of Illinois, Champaign, IL, USA
However, absence of explicit memory or an articulable representation of a stimulus does not necessarily mean that it went unprocessed, or even that it never reached conscious awareness: Stimuli might gain transient access to awareness but then be forgotten (inattentional amnesia; Wolfe, 1999), or a stimulus might be noticed but remain outside of semantic access (inattentional agnosia; Simons, 2000).

Although these paradigms might prove inadequate as exhaustive measures of explicit awareness (Holendar, 1986), they do permit tests of how much information is represented when stimuli fall outside of attention and when subjects cannot or do not report them. A variety of clever methods have emerged to reveal the subsequent impressions left by unnoticed stimuli, and the representations of those stimuli have been probed with methods such as subliminal learning (Rosenthal \& Humphreys, 2010) and negative priming (DeSchepper \& Treisman, 1996). Such tasks often use indirect measures of the influence of an unreported and unattended aspect of the display to show that those aspects have been processed. Such measures have revealed processing of global form information in the absence of attention: Low-level cues such as contours, surfaces, and Gestalt grouping are reliably processed without explicit awareness (Lamy, Segal, \& Ruderman, 2006; Mitroff \& Scholl, 2005; Moore, Grosjean, \& Lleras, 2003; Rosenthal \& Humphreys, 2010; Russell \& Driver, 2005). 
In a foundational experiment, Moore and Egeth (1997) used a cunning online measure of perceptual grouping. Their subjects judged the relative lengths of two lines that appeared superimposed on a background grid of black and white dots. Usually these dots were randomly colored and the lines differed in length. However, on some trials the lines were of equal length and the dots were colored in such a way that, when grouped, they formed a visual illusion that would cause one of the lines to look longer than the other. Subjects were systematically biased in their judgments of the line lengths in the direction consistent with the illusion, revealing the successful influence of the background. Despite the reliable effect of the illusion, few subjects reported noticing the pattern, and they did not select it reliably in a subsequent forced choice task. These results suggest that Gestalt grouping occurs automatically and does not require attention, but that the grouping patterns are not necessarily encoded into memory without attention. The absence of explicit memory for the patterns might explain why an earlier experiment that had used only explicit report to gauge representations of grouping had concluded that grouping does not occur without attention (Mack et al., 1992).

This sort of grouping in the absence of awareness might constitute implicit perception in the absence of attention and awareness. Several other studies, most of which are reported in Mack and Rock's (1998) seminal book on inattentional blindness, suggested that richer graphemic and semantic information might be processed, as well. In one such experiment, the subjects kept their eyes on a fixation point and judged which arm of a cross briefly presented in the periphery was longer. On the critical trial, a word replaced the fixation point while the cross was on screen. Most subjects did not report noticing the word, but they were more likely to complete a word stem with the word that had been presented than were control subjects who performed the stem completion task with no priming.

Related experiments have produced similar findings consistent with substantial perceptual processing prior to awareness. For example, people were more likely to notice their own name than a slightly distorted version of their name (e.g., "Jack" vs. "Jeck"), and they were more likely to notice a smiling emoticon than a frowning one. These results suggest that stimuli undergo extensive processing in the absence of attention, with selection and awareness occurring late in the processing stream; stimuli are filtered into awareness only after a detailed representation has been formed. Indeed, Mack and Rock (1998) noted that their series of experiments on implicit perception "are strong convergent evidence of late selection and extensive processing" (p. 181).

Any demonstration that unreported and unattended stimuli influence other aspects of perception would be consistent with late selection, but not all tests of this idea have been consistent with that conclusion. Mack and Rock (1998) also reported a collection of similar experiments in which people were not successfully primed to pick one interpretation of an ambiguous figure over another during inattentional blindness. Mack and Rock argued that the difference might result from words priming more strongly than pictures, but the varied performance is also consistent with sampling error, due to the relatively small numbers of subjects in each experiment. A largesample replication of their primary evidence for late selection would provide a more accurate estimate of the effect.

Collectively, these experiments suggest that a tremendous amount of information can be processed without attention, from basic contour and grouping information to semantic meaning. This information can both influence performance outside of awareness and prime subsequent performance on semantic tasks. Moore and Egeth's (1997) study and Mack and Rock's (1998) stem-completion study are perhaps the most prominent and important examples from the inattentional blindness literature of basic and complex information being processed without conscious perception or subsequent memory. In this registered report, we replicate these two classic findings using large-sample online studies. Online methods have reliably shown large inattentional blindness effects (e.g., Stothart, Boot, \& Simons, 2015; Ward \& Scholl, 2015; Wood \& Simons, 2017) and allow for rapid collection of large samples, making them especially well-suited to brief experiments with one critical trial.

\section{Experiment 1}

We attempted to replicate the primary finding from Moore and Egeth's (1997) study — that people will group the background dots and therefore be influenced by a visual illusion without awareness - with a modified paradigm. The original study differed from typical inattentional blindness paradigms in its trial structure. Usually, the subjects in an inattentional blindness experiment experience an unexpected object or event for the first time on the critical trial (e.g., Mack et al., 1992; Most et al., 2001; Simons \& Chabris, 1999). In this experiment, however, subjects performed an entire block in which half of the trials featured the pattern in the background, but they were not asked about the pattern until after its presentation in a second block of trials.

Our study used the same logic as the original one but adapted the procedure in order to adhere to a more typical inattentional blindness paradigm. The patterned dots appeared just once, in the final and critical trial, after which we probed awareness of the pattern. Rather than measuring the extent of illusion-induced bias for each subject (a within-subjects design), our large sample sizes collected via Mechanical Turk allowed us to calculate this measure between subjects (as is more typical of inattentional blindness studies). The original experiment also relied on the experimenter for both data 
collection and questioning of subjects, whereas ours was fully computerized. Moore and Egeth's (1997) primary finding was replicated in a study using a similar design (Lamy et al., 2006), but as in the original study, that replication study repeated the pattern in the background prior to the critical trial and relied on small sample sizes (nine and 16 subjects in the relevant experiments).

\section{Methods}

Subjects We aimed to collect usable data from 100 subjects per condition, for a target total of 400 . The subjects were USbased workers on Amazon Mechanical Turk. We checked worker IDs against a database of prior subjects using TurkGate (Goldin \& Darlow, 2013); anyone who had previously participated in an inattentional blindness experiment from our laboratory was informed that they were not eligible for this HIT ("human intelligence task," the term used for the tasks that workers complete on Mechanical Turk) and were excluded prior to participating.

The need for signed consent was waived by the institutional review board at the University of Illinois due to the low-risk nature of the experiment. Prior to the experiment, subjects were shown an information screen that provided experimenter and institutional review board contact information, explained that their responses would be anonymous, described how their data would be used, and noted that their participation was voluntary.

Subjects were automatically recruited in batches of up to nine and were randomly assigned to experimental conditions. On the basis of prior inattentional blindness studies using this approach to recruiting and testing subjects, we anticipated a roughly $35 \%$ exclusion rate, so we set a recruitment threshold of 600 subjects in order to end up with our target sample size of 400 after exclusions. No further batches were posted after this number was reached, and our final sample size was 621 subjects. Subjects received $\$ 0.25$ upon completing the experiment.

Materials and procedure A demonstration of the task, exactly as subjects experienced it but with no data collection, may be viewed at http://simonslab.com/rep/lines_demo.html.

The experiment was programmed entirely in Javascript (all materials may be found on the Open Science Framework, at https://osf.io/ $7 \mathrm{mtzn} /$ ). Upon loading the experiment, the script checked the display size of the device accessing it. If it detected a dimension of the display smaller than 480 pixels (the case with almost all smartphones), the experiment did not load, and subjects instead saw a screen asking them to reload the experiment on a device with a larger display.

At the start of the experiment, subjects were presented with an instruction screen explaining that they would be performing a difficult line judgment task, in which they would have to decide which of two very briefly presented lines was longer. Subjects then performed ten trials of the line judgment task (see Fig. 1). On each trial, a small black fixation cross (40 $\times 40$ pixels with three-pixel-thick lines) appeared for 1,000 $\mathrm{ms}$, followed by the line display for $200 \mathrm{~ms}$. The background of the line display consisted of a $28 \times 22$ grid of black and white dots on a medium gray (\#808080) field, $900 \times 600$ pixels. Each dot was 12 pixels in diameter, with 19 pixels between the centers of horizontally adjacent dots and 23 pixels between the centers of vertically adjacent dots. The entire dot grid measured $525 \times 495$ pixels.

On nonillusion trials, a randomly selected 49 dots (8\% of the dots in the grid) were colored black, while the rest were white. On illusion trials, a fixed number of dots were systematically colored black in order to create either the Ponzo or Müller-Lyer illusion with the lines. On trials featuring the Ponzo illusion, 20 dots were black. For the Müller-Lyer illusion, 24 dots were black.

Two parallel black lines, nine pixels thick, appeared horizontally centered in the dot array. The top line appeared approximately a third of the way down from the top, in the space between the sixth and seventh rows of dots, while the bottom line appeared roughly a third of the way from the bottom, between the 16th and 17th rows of dots. On nonillusion trials, one line was eight pixels longer than the other. The lengths were randomly selected from three possible pairs: 150 and 158 pixels, 153 and 161 pixels, or 156 and 164 pixels. The two lines in the pair were randomly assigned to the top and bottom positions.

On illusion trials, the lines were both 156 pixels long. Depending on the experimental condition, the illusion made either the top or the bottom line seem longer than the other line in the display. This marks a slight departure from the stimuli in the original study. Here we created both the Ponzo and MüllerLyer illusions on the same dot grid. In the original, Experiment 1 had used the Ponzo illusion on a smaller grid, and Experiment 3 had used the Müller-Lyer illusion on a larger grid. Rather than do two separate experiments, we adjusted the Ponzo illusion for the larger grid and added its two variants as an additional two conditions.

After the line display, a mask appeared for $1,000 \mathrm{~ms}$. The mask was a dot array, the same size as the one used during the line display, with 185 (30\%) of the dots randomly colored black. After the mask offset, subjects were asked to report which line, top or bottom, was longer.

Subjects completed nine nonillusion trials followed by one illusion trial. After making their line-length judgment for the final trial, subjects were asked whether they had noticed any pattern in the background of the dots. They were then asked to select the pattern they had seen from six images of dot grids without any lines, which featured the two variants of the Ponzo illusion, the two variants of the Müller-Lyer illusion, a random dot array, and a dot array with uniformly distributed 


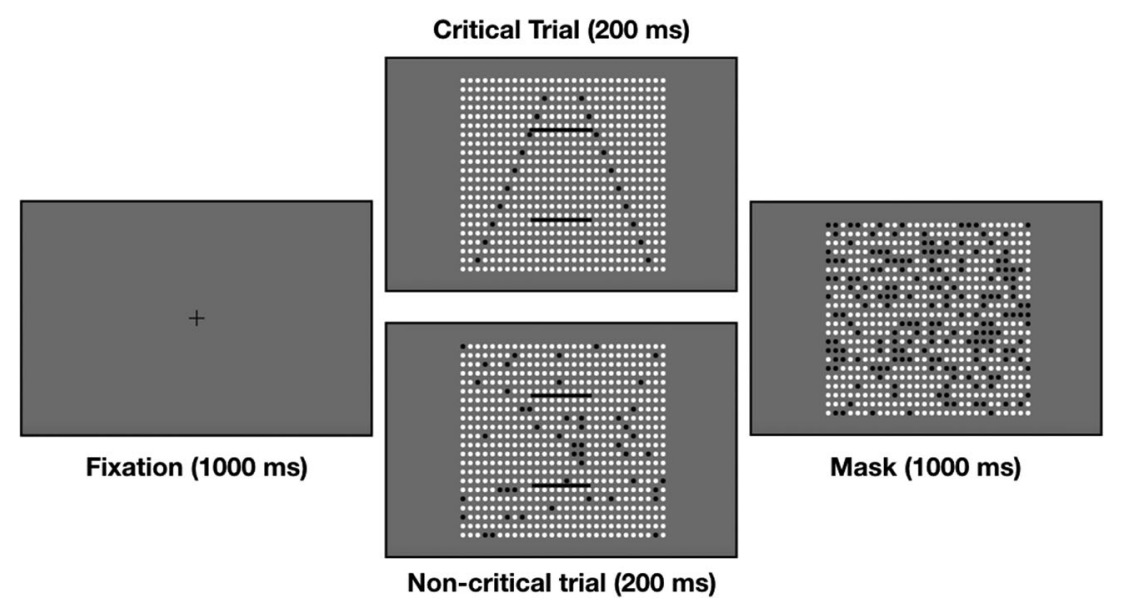

Fig. 1 Schematic of the procedure in Experiment 1. A fixation cross appears for 1,000 ms and is then replaced by the dot grid for $200 \mathrm{~ms}$. On noncritical trials, the dots are random and the two lines differ in length. On critical trials, the dots form one of four possible illusions

black dots (Fig. 2). They were also asked to rate their confidence in their selection as not at all confident, somewhat confident, or very confident.

Subjects were then presented with another screen, instructing them that for the final trial, they should ignore the lines and instead concentrate on the dots in the background. They were then presented with another illusion trial, identical to the inattention trial, and responded to the same forced choice question, which required them to select the pattern in the background from six images. The subjects then completed a brief survey that asked about their age, gender, vision correction, and prior experience with similar inattentional blindness studies.

After completing the survey, subjects were shown a debriefing screen that explained the purpose of the study. They clicked a button on this screen to get a completion code that they entered into Mechanical Turk in order to receive payment.

\section{Results and discussion}

All analyses were conducted using $\mathrm{R}$ version 3.5.1 ( $\mathrm{R}$ Core Team, 2018) and the packages ggplot2 version 2.2.1 (Wickham, 2009), dplyr version 0.7.4 (Wickham, Francois, Henry, \& Müller, 2017), purrr version 0.2.4 (Henry \& Wickham, 2017), tidyr version 0.8.0 (Wickham \& Henry, 2018), and stringr version 1.3.0 (Wickham, 2018).

Prior to the analysis, we excluded subjects who reported being younger than 18 years of age, who reported needing vision correction and not wearing it during the experiment, who reported prior experience with a similar task, or who selected the incorrect pattern following the full-attention trial. Exclusion rates were much higher than anticipated, primarily due to exclusions when subjects failed to correctly report the pattern on the full-attention trial; we excluded 361 subjects, or (two variants of the Ponzo illusion and two variants of the Müller-Lyer illusion) and the lines are of equal length. Immediately afterward, a random-dot mask appears for $1,000 \mathrm{~ms}$

$58 \%$ of our sample. Excluding these subjects guaranteed that the subjects in the analysis were capable of fully perceiving the patterns under conditions of full attention. Given that it was our preregistered exclusion plan, all statistics reported below are based on data after excluding these subjects; however, the overall pattern of results was the same, regardless of whether or not we excluded subjects on the basis of the fullattention trial (see Figs. 3, 4, and 5).

It is unclear why so many subjects failed the full-attention trial, particularly when Moore and Egeth's (1997) subjects had no difficulty on the full-attention trial. One contributor might have been the age of the subjects; our sample was older than the typical university sample, and exploratory analysis revealed that the older the subjects were, the less likely they were to pass the full-attention trial (although this does not fully account for the high failure rates). It is also possible that the instructions for the full-attention trial were not specific enough. Subjects were told to "ignore the lines and concentrate on the dots in the background," but not that they should keep their eyes fixed (even though the fixation cross appeared
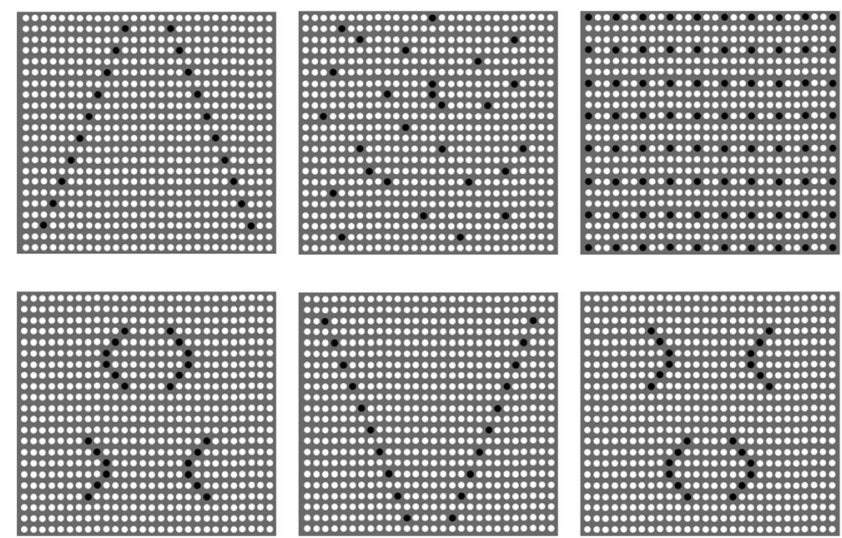

Fig. 2 The six dot patterns presented as alternatives for the forced choice recognition task 


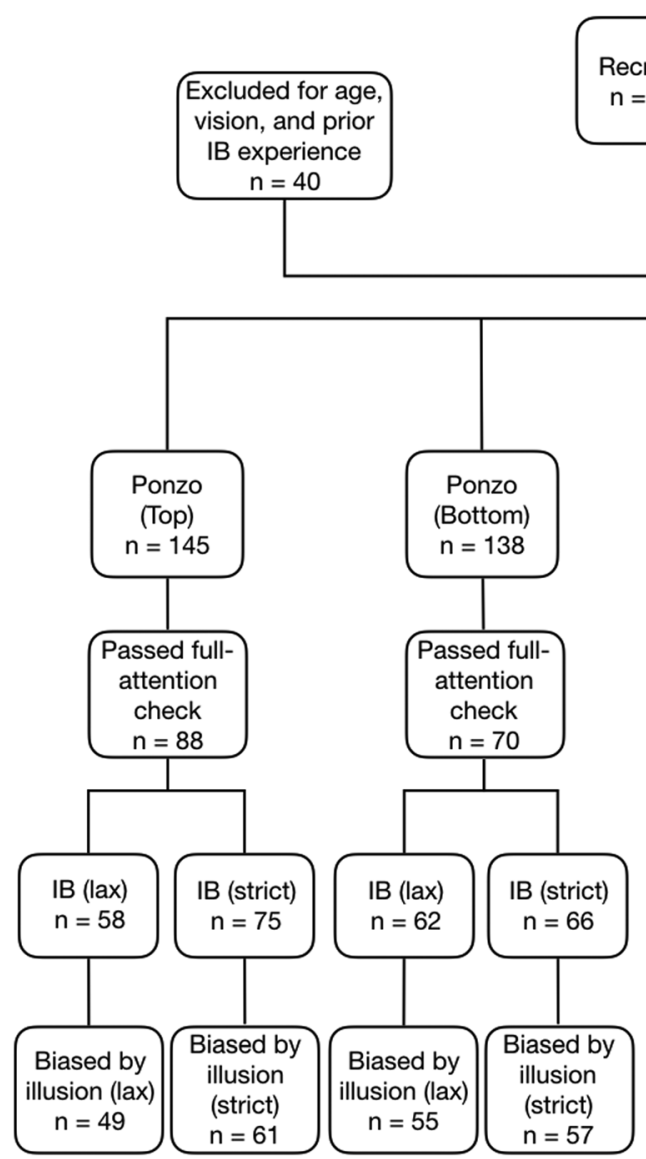

Fig. 3 Totals for subjects recruited, those excluded for vision and prior experience with inattentional blindness, subjects who passed the fullattention check, subjects who were inattentionally blind to the pattern on the critical trial, and subjects who selected the line consistent with

at the start of the trial). If subjects moved their eyes away from the center of the display in order to search the background dots, they could have missed the appearance of the pattern.

Line judgments on the critical trial were analyzed separately for noticers and nonnoticers who answered the fullattention check correctly. We used two different criteria to determine noticing: a lax criterion, in which subjects merely needed to report having noticed a pattern on the critical trial, and a strict criterion, in which subjects had to report noticing and also select the correct pattern on the forced choice task.

In all, 32\% (95\% CI: [26.2, 37.7]) of the subjects noticed a pattern on the critical trial by the lax criterion, and $16.5 \%$ (95\% CI: $[11.9,21.2])$ noticed by the strict criterion; noticing did not differ dramatically by illusion type (see Fig. 4). Among the subjects who did not report noticing the pattern in the background - the inattentionally blind subjects - the strengths of the illusion were comparable across illusion types, although the illusion was weaker for the Müller-Lyer variant in which the top line looked longer (Fig. 5). Overall, inattentionally blind subjects selected the illusion-consistent line as longer $84.2 \%$ (95\% CI: [78.5, 89.3]) of the time by the lax criterion, and $80.7 \%$ of the time (95\% CI: $[75.1,86.2])$
Recruited

$\mathrm{n}=621$

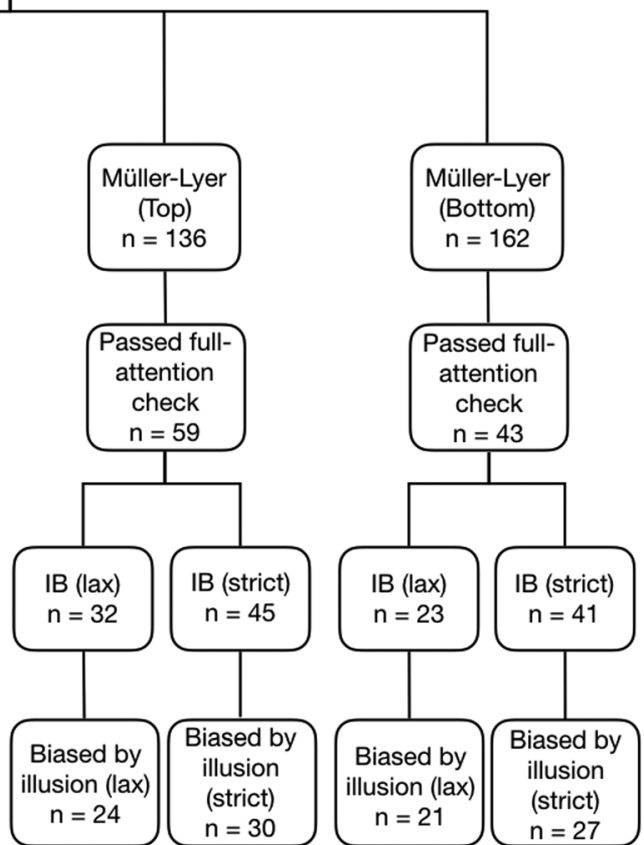

the illusion as being longer, broken down by condition. For a breakdown including subjects who failed the full-attention trial, see the supplementary material

by the strict criterion, where $50 \%$ would represent no effect of the illusion. This suggests that, consistent with the findings of the original study, subjects were influenced by the illusion when they did not notice it.

By comparison, of the noticers, by the lax criterion $74.7 \%$ (95\% CI [65.1, 84.3]) judged the line consistent with the illusion to be longer, and by the strict criterion $83.7 \%(95 \% \mathrm{CI}$ $[72.1,93])$ did so.

The illusion influenced judgments of line length just as strongly, regardless of whether or not subjects reported noticing the background. Consistent with Moore and Egeth's (1997) findings, our study found evidence of the influence of perceptual grouping under conditions of inattentional blindness.

\section{Experiment 2}

In Experiment 2, we aimed to reproduce the primary finding of Mack and Rock's (1998) classic stem completion experiment. We used a procedure similar to that of the original study, although in this variant subjects perform five noncritical trials, one critical trial, and one full- 


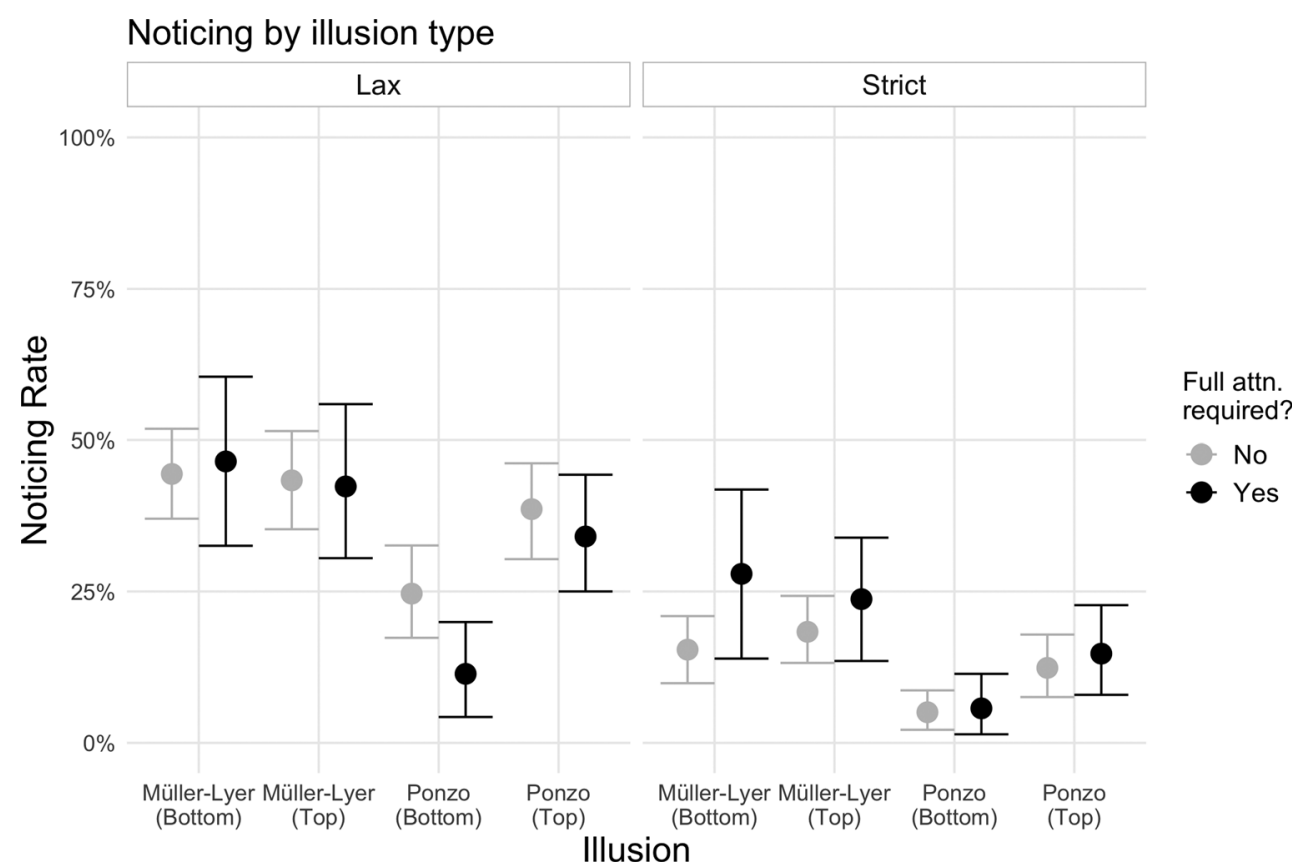

Fig. 4 Noticing rates for each of the four illusion variants, broken down by noticing criterion. Lax noticers needed only to report noticing a pattern in the dots on the critical trial; strict noticers had to report having noticed a pattern and correctly identified it in a forced choice task. Error bars show
95\% bootstrapped confidence intervals. The black points exclude subjects who responded incorrectly following the full-attention trial. The gray points include these subjects

The original findings are broken down into raw numbers in Table 1. In this replication we used a much larger sample to get a more reliable measure of the effect. attention trial; there were no divided-attention trials. Additionally, the original study had had 20 people assigned to each of four priming words, for 80 total.

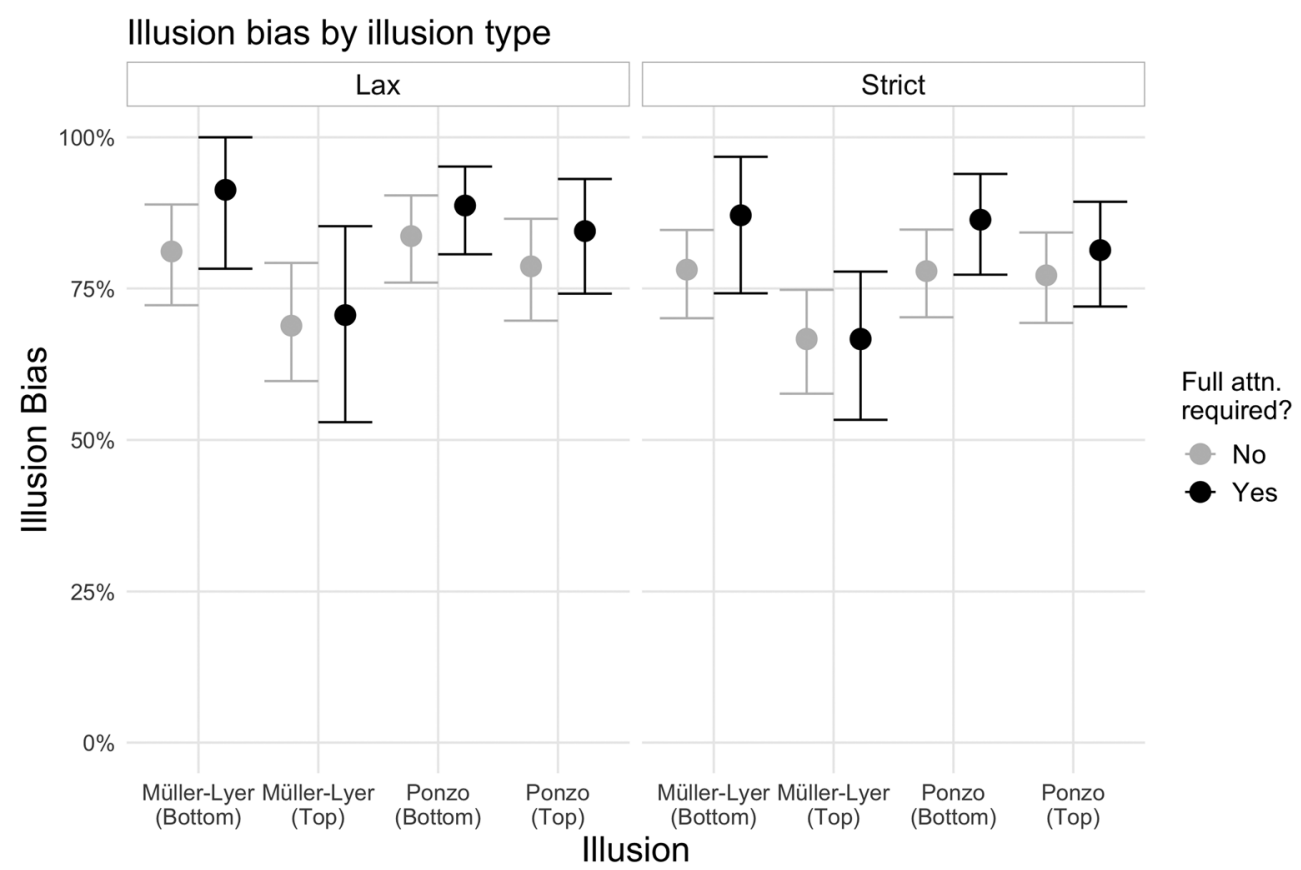

Fig. 5 Rates at which nonnoticers judged the line consistent with the illusion to be longer when both lines were of equal length, broken down by illusion type and noticing criterion. Lax noticers needed only to report noticing a pattern in the dots on the critical trial; strict noticers had to report having noticed a pattern and correctly identify it in a forced choice task. Error bars show 95\% bootstrapped confidence intervals. The black points exclude subjects who responded incorrectly following the full-attention trial. The gray points include them. 
Table 1 Numbers reported by Mack and Rock (1998) of subjects who were inattentionally blind (IB), of IB subjects who completed one of the two stems with the critical word, and of control subjects who provided the critical word as one of their two responses to a stem completion task

\begin{tabular}{llll}
$\begin{array}{l}\text { Critical } \\
\text { Word }\end{array}$ & $\begin{array}{l}\text { IB } \\
\text { Subjects }\end{array}$ & $\begin{array}{l}\text { IB Subjects Completing } \\
\text { Stem With Critical Word }\end{array}$ & $\begin{array}{l}\text { Control Subjects } \\
\text { Completing Stem } \\
\text { With Critical Word }\end{array}$ \\
\hline Flake & $13 / 20$ & $7 / 13$ & $2 / 20$ \\
Grace & $12 / 20$ & $3 / 12$ & $0 / 20$ \\
Short & $14 / 20$ & $4 / 14$ & $3 / 20$ \\
Prize & $11 / 20$ & $4 / 11$ & $1 / 20$ \\
\hline
\end{tabular}

\section{Method}

Subjects We aimed to collect usable data from 50 subjects per prime word, for a target total of 350 subjects. In anticipation of a roughly $35 \%$ exclusion rate, we set a recruitment threshold of 540 subjects and recruited 557 in total to finish with our desired postexclusion sample size. Otherwise, the subject characteristics and recruiting procedure were identical to those of Experiment 1.

Selecting prime words In describing their prime-word selections, Mack and Rock (1998) stated: "In order for this procedure to permit this inference, the prime must be chosen so that its stem can be completed by many other common English words. Moreover, the prime word must be among the most unlikely or infrequent of them, otherwise word frequency alone might account for its choice" (p. 176). Their method of choosing their particular primes is not described.

To select our prime words, we downloaded the 5,000 most common words in the English language according to the COCA corpus (Davies, n.d.) and extracted the seven most common twoletter stems for all five-letter words. These were $s t-, s h$-, $t r-, c h$-, $c l-, d r-$, and $g r$ - Then, again using the COCA database, we selected as our prime words the 50th most common five-letter word that had this stem. Shank, chafe, and grown met this criterion unambiguously. For the other stems, the 50th most common word had both a noun form and a verb form on the list; this word was still selected if the noun and verb were both within the 40th50th most common words. This applied to stall (noun form at $\# 48$, verb form at \#50) and trawl (noun form at \#42 and verb form at \#50). For $c l$-, the repeats for \#50 were too far apart, so we chose cleft (noun form at \#44, adjective form at \#51). In the case of dreck, we went to \#51 because the 50th word, droit, is typically seen only in French phrases, according to the usage data from COCA.

Materials and procedure A demonstration of the task, exactly as subjects experienced it but with no data collection, may be viewed at http://simonslab.com/rep/cross_demo.html. As with Experiment 1, the script checked for small displays before loading.
Subjects first saw an instruction screen explaining that they would be performing a difficult line judgment task, and that they would have to decide which arm of a cross was longer after viewing it very briefly. Subjects then proceeded to six trials of the line judgment task (see Fig. 6).

At the start of each trial, a small fixation dot (ten-pixel diameter) appeared in the center of a circular border (500 pixels in diameter and three pixels thick) for $1,000 \mathrm{~ms}$. On the noncritical trials, a cross then appeared for $200 \mathrm{~ms}$. The cross appeared at one of four possible peripheral locations (determined randomly between subjects but fixed for a given subject), displaced 100 pixels vertically and 100 pixels horizontally from fixation. The lines of the cross were two pixels thick, and the two arms were randomly selected without replacement from four possible lengths (135, 165, 195, and 225 pixels). The minimum difference in length between the two arms was therefore 30 pixels, and the maximum was 90 pixels. The longer arm was randomly assigned to be either the horizontal or the vertical arm on each trial.

After $200 \mathrm{~ms}$, the cross and fixation dot (or word, on critical trials) offset while the circular border remained onscreen for another 1,000 ms. After the border offset, subjects were asked which arm of the cross was longer, the vertical (topbottom) arm or the horizontal (left-right) arm.

After subjects had made their line judgment on the critical trial, they performed a stem completion task. They were presented with the first two letters of the prime word, followed by three underscores (e.g., G R _ _ ). The word updated as they typed into a text box, ignoring spaces and special characters. The display word only showed the first three letters entered. Subjects were free to delete input and start over, and they submitted their response by clicking a button. Inputs shorter than five letters (including the stem) were not accepted, and subjects were informed that they had to fill in all of the blanks before they could proceed.

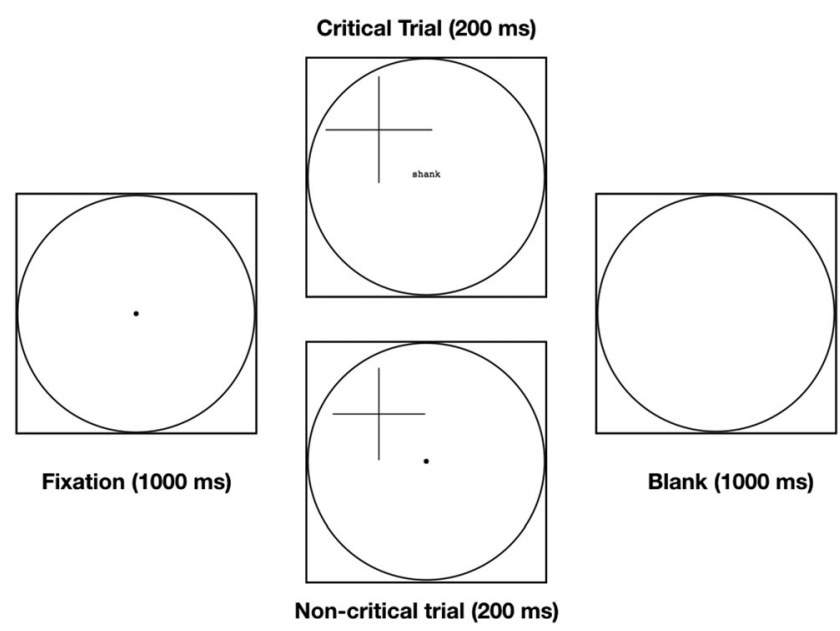

Fig. 6 Schematic of the procedure in Experiment 2. The fixation dot appeared for $1,000 \mathrm{~ms}$, followed by the cross for $200 \mathrm{~ms}$. On the critical trial, the prime word replaced the fixation dot at the moment of cross onset. Cross and fixation dot then both offset for $1,000 \mathrm{~ms}$ 
Following the stem completion, subjects were asked whether they had noticed a word appearing where the fixation dot had normally appeared on the preceding trial. They were then asked to select the word from a list of five possibilities and to rate their confidence in their choice (not at all confident, somewhat confident, or very confident). For the filler words in the forced choice recognition question, all of the options completed the same two-letter stem as the prime word. They were selected to be (roughly) the most common and the 11th, 21st, and 31 st most common words that completed the stem (of all lfive-etter words completing the stem). If the next candidate word did not have at least two letters different from the previous word as counted by letter position (e.g., for "grave" and "grade," only the fourth letter differs), the next-most-common word was selected instead.

Following the critical trial, subjects were instructed to ignore the cross on the next trial and to instead focus on the fixation dot in the center. Subjects were then presented with another critical trial, with the prime word replacing the fixation dot when the cross onset. Subjects then completed the same forced choice recognition question that had followed the inattention trial and were given the same demographic questions as in Experiment 1 before reading a debriefing screen and receiving their completion codes.

\section{Results and discussion}

Analyses were conducted with the same software as in Experiment 1. Prior to the analysis, we excluded subjects who reported being younger than 18 years of age, who reported needing vision correction and not wearing it during the experiment, who reported previous experience with inattentional blindness experiments, or who failed to choose the prime word following the full-attention trial. We excluded 109 subjects, or 19.6\% of our sample. As in Experiment 1, the conclusions did not change, regardless of whether the fullattention criterion was used to exclude subjects (see Figs. 7, 8, and 9).

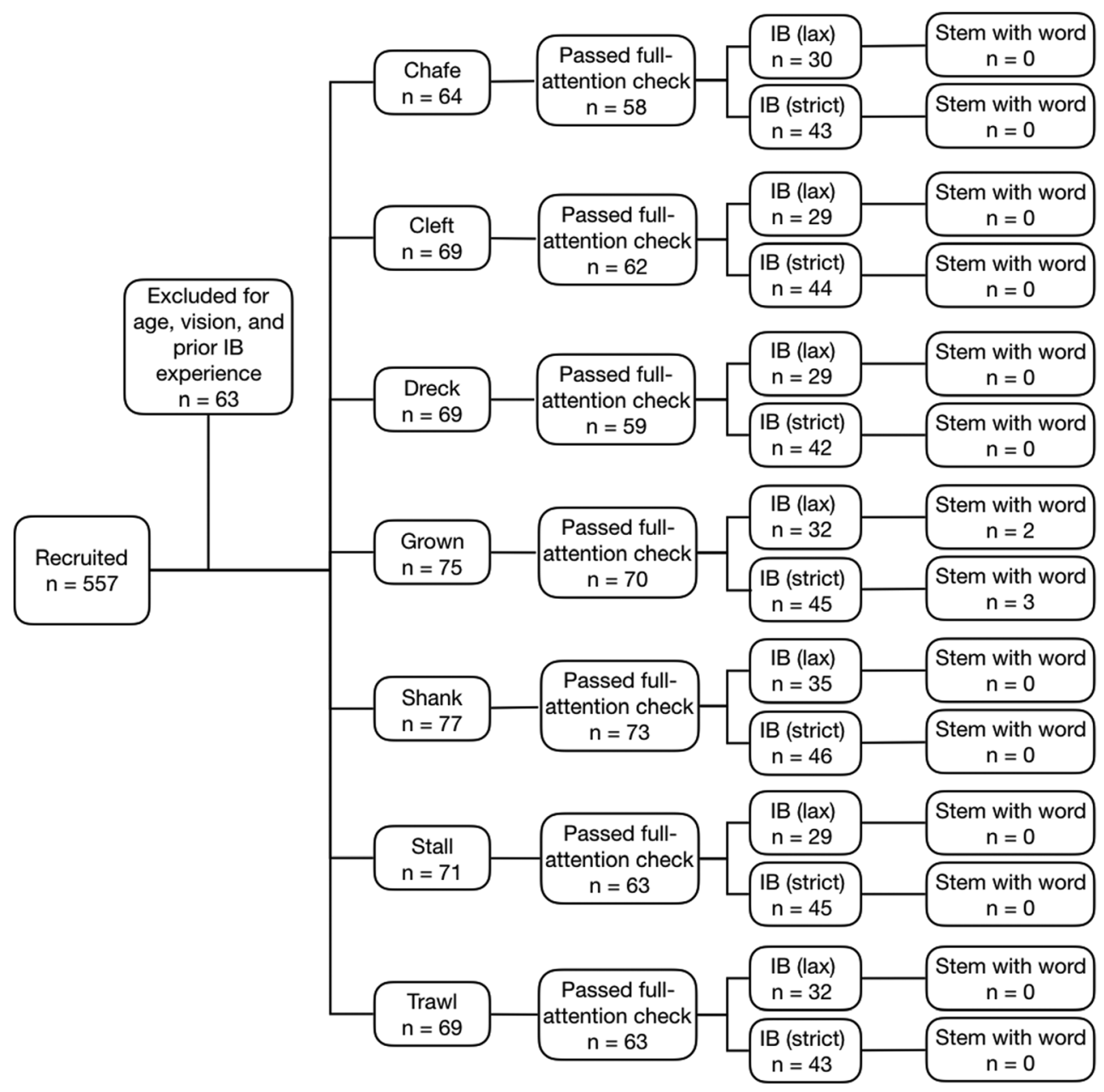

Fig. 7 Totals for subjects recruited, those excluded for vision and prior experience with inattentional blindness, subjects who passed the fullattention check, subjects who were inattentionally blind to the prime word on the critical trial, and inattentionally blind subjects who completed the stem with the prime word, broken down by prime word. For a breakdown including subjects who failed the full-attention trial, see the supplementary material 
We used two different criteria for determining whether a subject had noticed the word on the critical trial. For the laxer criterion, a subject simply had to report seeing the word to be counted as having noticed it. For the stricter criterion, subjects had to both report having seen the word and select the correct word from the forced choice recognition task. Overall, 51.8\% (95\% CI: $[47.1,56.3])$ of subjects noticed the word on the critical trial by the lax criterion, and $31.3 \%$ (95\% CI: [27.2, 35.9]) did so by the strict criterion. Noticing rates did not vary by prime word (Fig. 8).

A subject was classified as having supplied the critical word on the stem completion in cases of perfect matches only; we did not attempt to take misspellings into account. Using this criterion, $0.9 \%$ (95\% CI: [0.0, 2.3]) of the lax nonnoticers supplied the prime word on the stem completion task, whereas $32.8 \%$ (95\% CI: [26.7, 39.2]) of the lax noticers did, and $1.0 \%$ (95\% CI: [0.0, 2.3]) of the strict nonnoticers offered the prime word, whereas $53.6 \%$ (95\% CI: [45.7, 62.1]) of the strict noticers did (see Fig. 9). The rates of stem completion with the prime word did not vary according to the word itself.

Inattentionally blind subjects almost never completed the stem with the prime word, suggesting that they did not process the word without conscious awareness of it to a degree that resulted in priming on the word-stem completion task. Although many subjects who noticed the prime completed the word stem with the prime word, unlike Mack and Rock (1998), we found no evidence for word-stem priming under conditions of inattentional blindness.

\section{General discussion}

How fully do we perceive and process information that we do not consciously report? Moore and Egeth (1997) showed that grouping influenced perception under conditions of inattentional blindness, and Mack and Rock (1998) reported evidence for semantic priming by unreported words. These studies are among the most importing findings in the inattentional blindness literature, revealing evidence that stimuli are encoded and processed even when they are not consciously detected. In two large-sample, preregistered online experiments, we attempted to replicate these two classic findings.

In our re-creation of Moore and Egeth's (1997) experiment, subjects who were inattentionally blind to the pattern formed by the dots in the critical trial were nevertheless reliably influenced by the resultant illusion. This large effect-estimated at an $80 \%$ illusion bias between subjects - was robust to illusion type and to the choice of inclusion/exclusion criteria. Some variants of the illusions were slightly more likely to be noticed, and they varied somewhat in the strength of the illusion induced, but in all cases the pattern was consistent with Moore and Egeth's findings.

In our version of Mack and Rock's (1998) experiment, only three subjects out of 308 nonnoticers completed the stem with the prime word. All three completed the stem $g r$ - with grown; no subject completed any other stem with the primed word. When subjects noticed the prime word on the critical trial,

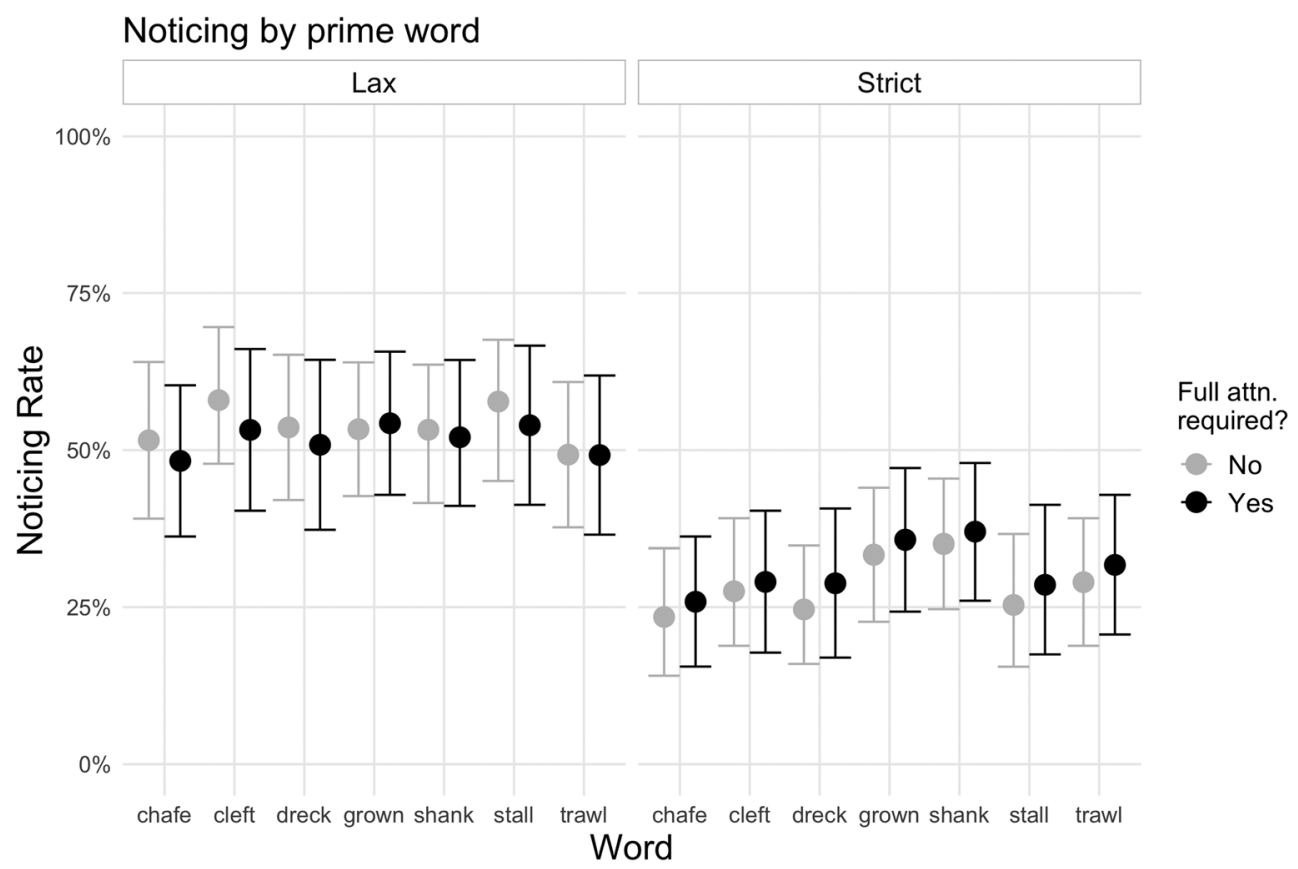

Fig. 8 Noticing rates for each of the seven possible priming words, broken down by noticing criterion. Lax noticers needed only to report noticing a word on the critical trial; strict noticers had to report having noticed a word and select it in a forced choice task. The black points exclude subjects who responded incorrectly following the full-attention trial. The gray points include them. On critical trials, the fixation dot was replaced by the priming word when the cross onset, leaving the word visible for $200 \mathrm{~ms}$. The letters were 20 pixels in height, and the word spanned 60 pixels in width. The letters appeared in Courier font, which is monospace; thus, every word had the same width 


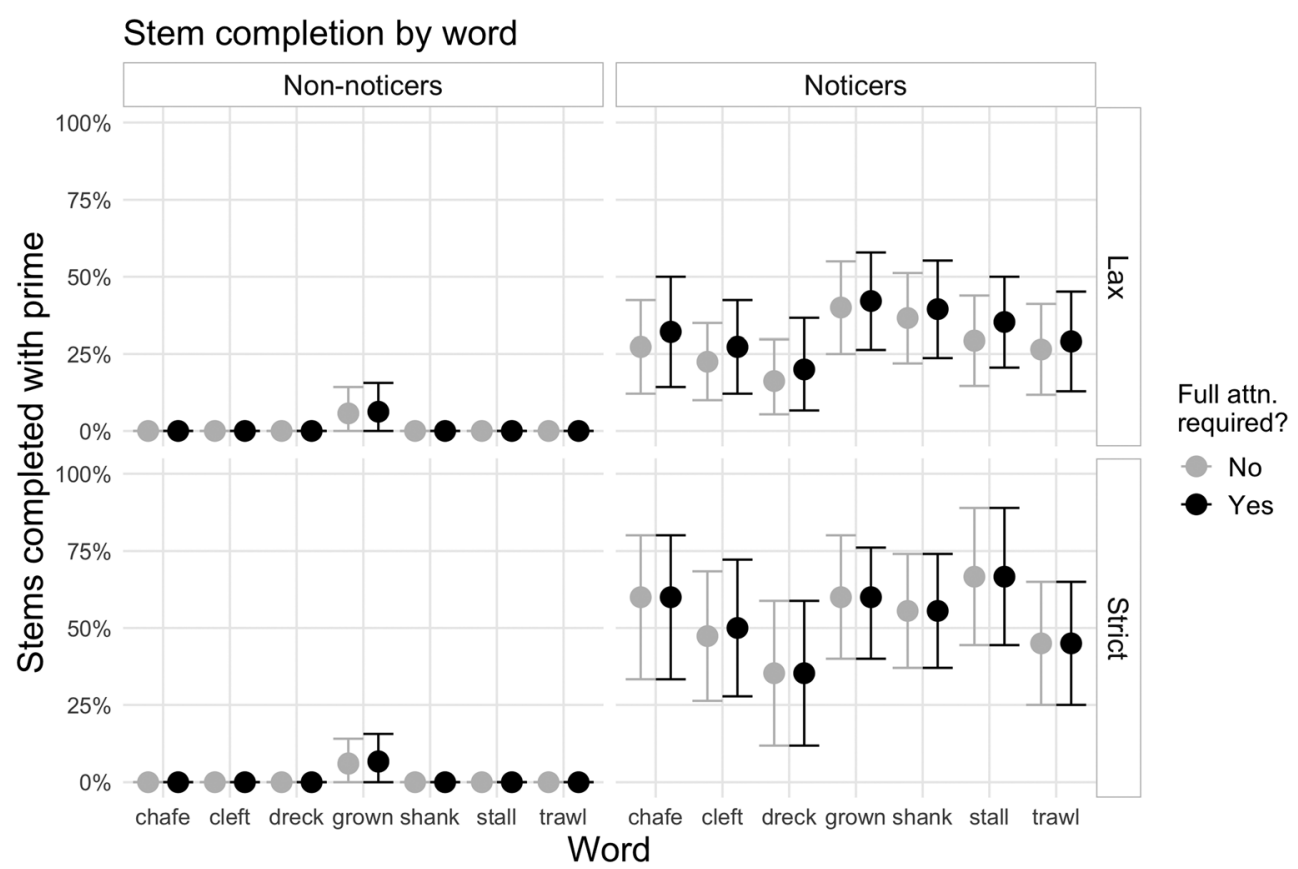

Fig. 9 Rates at which noticers and nonnoticers completed the stem with the priming word, broken down by word and noticing criterion. Lax noticers needed only to report noticing a word on the critical trial; strict noticers had to report having noticed a word and select it in a forced choice task. The black points exclude subjects who responded incorrectly following the full-attention trial, whereas the gray points include them

study was in the choice of prime words. We deliberately selected stems that would maximize the number of common words that completed them, and selected prime words that are used extremely infrequently - so much so that they should almost never be offered as stem completions by chance. It is possible that the magnitude of priming in this context was too small to prime such rare words, and priming is only strong enough to prime stem completion if there is a nonzero chance of offering the word without priming. That said, the difference between the priming condition and the control condition in the original study was small; an average of three more subjects completed each stem with the primed word in the prime conditions than in the control conditions.

We expect the pattern of results we observed to be robust to minor changes in display types or stimuli. Inattentional blindness effects have been elicited in both controlled laboratory settings and less controlled, nonlab situations (e.g., science museums; Cartwright-Finch \& Lavie, 2007), so we have reason to believe that these results should generalize to other data collection methods and settings.

Author note K.W. is supported by an NSF Graduate Student Fellowship. K.W. and D.J.S. jointly planned and designed the experiments. K.W. programmed the experiments and analyses, oversaw data collection, generated the figures, and drafted the manuscript. K.W. and D.J.S. critically edited the manuscript. Both authors approved the final version for submission. 
Publisher's Note Springer Nature remains neutral with regard to jurisdictional claims in published maps and institutional affiliations.

\section{References}

Cartwright-Finch, U., \& Lavie, N. (2007). The role of perceptual load in inattentional blindness. Cognition, 102, 321-340. https://doi.org/10. 1016/j.cognition.2006.01.002

Davies, Mark. (n.d.) The Corpus of Contemporary American English (COCA): 560 million words, 1990-present. Available online at https://corpus.byu.edu/coca/

DeSchepper, B., \& Treisman, A. (1996). Visual memory for novel shapes: Implicit coding without attention. Journal of Experimental Psychology: Learning, Memory, and Cognition, 22, 27-47. https:// doi.org/10.1037/0278-7393.22.1.27

Goldin, G., \& Darlow, A. (2013). TurkGate (Version 0.4.0) [Software]. Available from http://gideongoldin.github.com/TurkGate/

Henry, L., \& Wickham, H. (2017). purrr: Functional programming tools (R package version 0.2.4). Retrieved from https://CRAN.R-project. org/package $=$ purrr

Holendar, D. (1986). Semantic activation without conscious identification in dichotic listening, parafoveal vision, and visual masking: A survey and appraisal. Behavioral and Brain Sciences, 9, 1-23, disc. 2366. https://doi.org/10.1017/S0140525X00021269

Lamy, D., Segal, H., \& Ruderman, L. (2006). Grouping does not require attention. Perception \& Psychophysics, 68, 17-31.

Mack, A., \& Rock, I. (1998). Inattentional blindness. Cambridge: MIT Press.

Mack, A., Tang, B., Tuma, R., Kahn, S., \& Rock, I. (1992). Perceptual organization and attention. Cognitive Psychology, 24, 475-501.

Mitroff, S. R., \& Scholl, B. J. (2005). Forming and updating object representations without awareness: Evidence from motion-induced blindness. Vision Research, 45, 961-967. https://doi.org/10.1016/j. visres.2004.09.044

Moore, C., Grosjean, M., \& Lleras, A. (2003). Using inattentional blindness as an operational definition of unattended: The case of surface completion. Visual Cognition, 10, 299-318. https://doi.org/10.1080/ 13506280143000041

Moore, C. M., \& Egeth, H. (1997). Perception without attention: Evidence of grouping under conditions of inattention. Journal of Experimental Psychology: Human Perception and Performance, 23, 339-352. https://doi.org/10.1037/0096-1523.23.2.339

Most, S. B., Simons, D. J., Scholl, B. J., Jimenez, R., Clifford, E., \& Chabris, C. F. (2001). How not to be seen: the contribution of similarity and selective ignoring to sustained inattentional blindness.
Psychological Science, 12, 9-17. https://doi.org/10.1111/14679280.00303

Neisser, U., \& Becklen, R. (1975). Selective looking: Attending to visually specified events. Cognitive Psychology, 7, 480-494. https://doi. org/10.1016/0010-0285(75)90019-5

R Core Team. (2018). R: A language and environment for statistical computing (Version 3.5.1). Vienna: R Foundation for Statistical Computing. Retrieved from https://www.R-project.org/

Rosenthal, O., \& Humphreys, G. W. (2010). Perceptual organization without perception: The subliminal learning of global contour. Psychological Science, 21, 1751-1758. https://doi.org/10.1177/ 0956797610389188

Russell, C., \& Driver, J. (2005). New indirect measures of "inattentive" visual grouping in a change-detection task. Perception \& Psychophysics, 67, 606-623. https://doi.org/10.3758/BF03193518

Simons, D. J. (2000). Attentional capture and inattentional blindness. Trends in Cognitive Sciences, 4, 147-155. https://doi.org/10.1016/ S1364-6613(00)01455-8

Simons, D. J., \& Chabris, C. F. (1999). Gorillas in our midst: Sustained inattentional blindness for dynamic events. Perception, 28, 1059 1074. https://doi.org/10.1068/p2952

Stothart, C. R., Boot, W. R., \& Simons, D. J. (2015). Using Mechanical Turk to assess the effects of age and spatial proximity on inattentional blindness. Collabra: Psychology, 1(1), 2:1-7. https:// doi.org/10.1525/collabra.26

Ward, E. J., \& Scholl, B. J. (2015). Inattentional blindness reflects limitations on perception, not memory: Evidence from repeated failures of awareness. Psychonomic Bulletin \& Review, 22, 722-727. https:// doi.org/10.3758/s13423-014-0745-8

Wickham, H. (2009). ggplot2: Elegant graphics for data analysis. New York: Springer.

Wickham, H. (2018). stringr: Simple, consistent wrappers for common string operations (R package version 1.3.0). Retrieved from https:// CRAN.R-project.org/package $=$ stringr

Wickham, H., Francois, R., Henry, H., \& Müller, K. (2017). dplyr: A grammar of data manipulation ( $\mathrm{R}$ package version 0.7 .4 ). Retrieved from https://CRAN.R-project.org/package=dplyr

Wickham, H., \& Henry, L. (2018). tidyr: Easily tidy data with "spread()" and "gather()" functions (R package version 0.8.0). Retrieved from https://CRAN.R-project.org/package=tidyr

Wolfe, J. M. (1999). Inattentional amnesia. In V. Coltheart (Ed.), Fleeting memories: Cognition of brief visual stimuli (pp. 71-94). Cambridge: MIT Press.

Wood, K., \& Simons, D. J. (2017). Selective attention in inattentional blindness: Selection is specific but suppression is not. Collabra: Psychology, 3(1), 19. https://doi.org/10.1525/collabra.90 